\title{
Underwater Image Enhancement using Improved Bat Algorithm
}

\author{
Mayuresh Deshmukh \\ Masters in computer science \\ Illinois Institute of Technology, Chicago, US \\ mayurdeshmukh2442@gmail.com
}

\begin{abstract}
From the past decades, improving the superiority of an Underwater Image (UI) has received well recognition in terms of the deprived image visibility that is occurred due to the water medium physical property. The light absorption of wavelength-reliant, as well as scattering in an underwater view, degrades the image visibility which causes lesser contrast as well as distorted color casts. To overcome the issues, a highly developed technique is adopted for the advancement and it improves the UI classification. Specifically, by exploiting the Improved Bat Algorithm (IBA), the underwater input image RGB is enhanced. The next stage is feature extraction. To the enhanced Principle Component Analysis (PCA) technique, the extracted attributes are given as input; here the dimensions of the features are minimized. Subsequently, the classification operation is carried out by exploiting the ANFIS classifier. Finally, the classified improved deeper water images besides the improved shallow water images which are seen are available in the testing stage. Finally, the simulation outcomes for the proposed and conventional methods are analyzed. The developed UIE system shows superior accuracy while compared with the conventional techniques.
\end{abstract}

Keywords: ANFIS Classifier, Classification, Feature Extraction, PCA,Underwater Image

\begin{tabular}{ll} 
Nomenclature & \\
\hline Abbreviations & Descriptions \\
\hline PSO & Particle Swarm Optimization \\
DNN & Deep Neural Network \\
IFM & Image Formation Model \\
PCA & Principal Component Analysis \\
UISA & Underwater Image Synthesis Algorithm \\
SUID & Synthetic Underwater Image Dataset \\
CFA & Color Filter Array \\
MR & McCann Retinex \\
UIE & Under-Water Image Enhancement \\
\hline
\end{tabular}

\section{Introduction}

In ocean resources exploration as well as engineering, underwater vision plays a significant task. Because of the selective light absorption as well as wavelength-dependent UI forever undergoes bluish as well as color castes. From the imaging scene while the distance to the camera is maximized, initially the red channel is disappeared. The map of the red channel is darkened as well as the pixels value in the red channel turns out to be small. Regarding, such image color must be rectified. In addition, the light scattering creates the contrast relatively minimum. Therefore, the UI contrast is frequently unacceptable [1].

The UI range is increased using artificial light sources that are frequently exploited. Still, using the scattering as well as absorption, the artificial light is affected badly. Meanwhile, non-uniform illumination is developed ensuing in intense spots at the UI center with inadequate illumination to the boards. For example, another quality degradation phenomenon involves shadowing. Therefore, the valuable information extraction for underwater scenes needs effectual techniques to rectify the color, enhance the clarity and identify blurring as well as background scattering that is the aspire of image 
restoration as well as enhancement techniques [8], [9]. Because of the complex underwater environment, these are specifically confronted, wherein degrading of images are occurred by the manipulation of absorption of light, water turbidity, as well as scattering that might alter extensively [2].

Various improvement applications were developed to enhance the underwater degraded image visibility namely Retinex based algorithms, histogram equalization, PSO, and fusion-based algorithms. Not like improvement strategies, restoration techniques are basically dedicated to recovering the original reflection characteristics of the scene based on the UI degradation technique. The cross-domain, as well as cross-view image matching, is not considered as an insignificant issue. Even though this issue is alleviated by prognostic both images of the surface water in the horizontal plane, the UI features do not unswervingly go with their aerial equivalent. The top view is captured by the aerial images when the front view of the scene is captured by the underwater acoustic images. One more issue is the water bodies' detection, objects, and structures in aerial images due to the texture as well as color change according to the season and weather of the year [4].

The main contribution of this paper is to propose a highly developed model for the development and it enhances the UI classification. Purposely, by exploiting the IBA, the underwater input image RGB is enhanced. The next stage is feature extraction. To the enhanced PCA model, the extracted attributes are given as input. At last, the experimentation results for the proposed and conventional methods are analyzed.

\section{Literature Review}

In 2020, Matheus Machado Dos Santos et al [1], developed a cross-view as well as cross-domain image identical which was exploited by the underwater acoustic image as well as a color aerial image to recognize the given images are captured or not in a similar place. In partially structured environments, the technique was modeled to match the images obtained with share features namely marinas as well as harbors. The pipeline integrates the conventional image processing techniques as well as DNN approaches.

In 2020, Changli Li et al [2], presented a new color correction approach based on CFA. Moreover, they had also presented a developed approach on the basis of the Retinex with dense pixels as well as the transformation of the adaptive linear histogram for degraded color-biased UI. In the RGB space, for any digital image that was captured using the digital camera exploiting the CFA, due to the interpolation procedure their RGB values were coupled as well as a dependent. Therefore, to compensate for attenuation of the red channel, they tried to attempt from the green and blue channel underwater degraded images. To effectively handle the blurred as well as minimum brightness images, the Retinex technique was extensively exploited. To choose a spiral path to calculate the illumination, for the pixel comparison the MR technique was exploited.

In 2017, Yan Wang et al [3], worked on image restoration as well as enhancement techniques that handle general UI impairments such as tackle a few severe distortions as well as degradations. Initially, the main reason behind the quality minimization in UI regarding the underwater IFM was introduced. Subsequently, the underwater restoration techniques taking into consideration of IFM free as well IFM based techniques were reviewed.

In 2019, Miao Yang et al [4], worked on underwater image processing as well as intelligent applications. So they had reviewed more than 120 research was performed on the basis of the current progression in UI enhancement as well as restoration. Here, the techniques include available codes, datasets as well as conventional techniques restrictions to make possible the wide-ranging analysis of UI enhancement as well as restoration. Moreover, the detailed analysis and evaluation were performed on the basis of the corresponding techniques on five kinds of underwater cases that examine the technique's applicability in diverse underwater circumstances.

In 2019, Guojia Hou et al [5], modeled an UISA, in that based upon the real-world UI, from an outdoor ground truth image has been produced a synthetic UI. A novel large-scale benchmark was established on the basis of the scheme which comprises synthetic UI as well as ground truth images of a similar scene named SUID. Based on the underwater IFM the SUID model was designed and the distinctiveness of underwater optical possessing, propagation, solid reliability, and feasibility.

\section{Developed Approach for Under Water Image}

Image enhancement is a technique that includes input image processing so that it is very clearly evident for the appropriate applications. The developed model presents a novel technique to enhance the low contrast images. The green and blue are superior in UI, hence the channels need to be enhanced. The developed technique comprises 4 stages. At first, the image's RGB values are changed so that the 
enhanced content in the image is increased. This process is achieved by the proposed optimization approach by exploiting the fitness model. Subsequently, the Harris, SURF, Hurst exponent, Skewness Mean, kurtosis, SD, contrast, entropy, correlation, and homogeneity features are extracted. By exploiting EPCA, the dimensionality of features is abridged. Here, the ANFIS classifier is exploited for the classification. The UI is classified into shallow images and as well as deep water images centered on the features are also extracted. Fig 1 demonstrates the architecture model of the proposed approach.

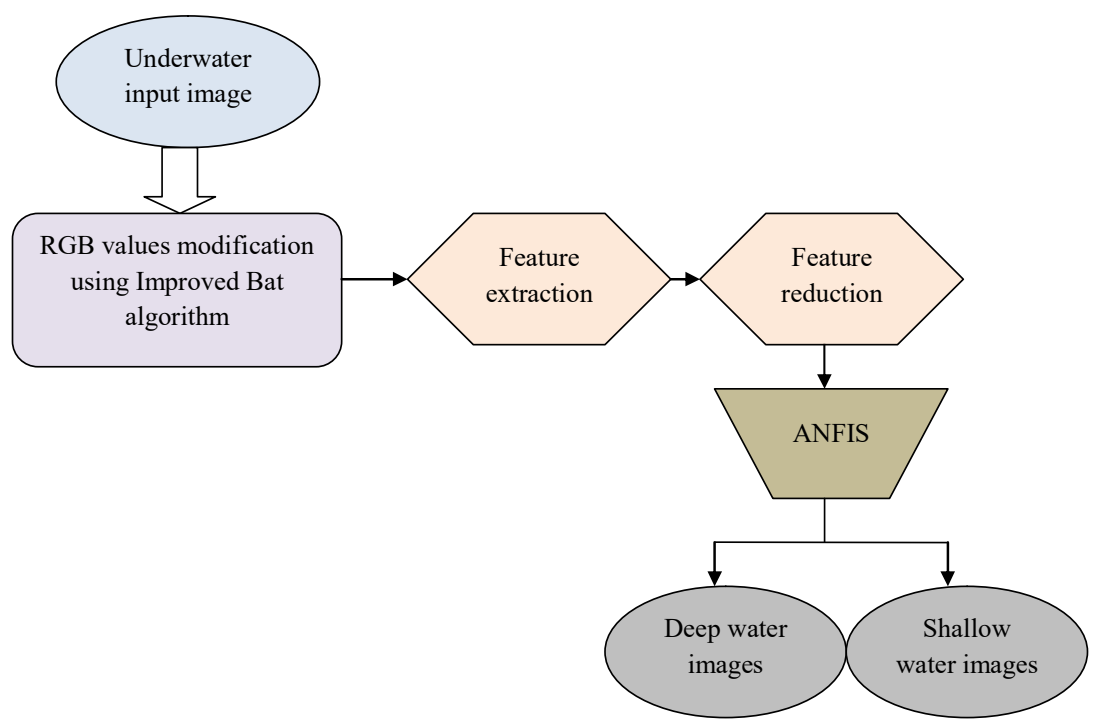

Fig. 1. Architecture model of the developed technique

\section{a) Input Image}

At first, eq. (1) indicates the adopted UI will be, wherein, $U_{m}$ indicates given underwater input image and $Z$ indicates the aggregate count of images on the database $D$. From the database, $D$ these numbers of UI are USED in the improvement and classification procedure.

$$
\mathrm{U}_{\mathrm{m}}=\left\{\mathrm{U}_{\mathbf{1}}, \mathrm{U}_{\mathbf{2}}, \mathrm{U}_{\mathbf{3}} \ldots \ldots \ldots \mathrm{U}_{\mathrm{n}}\right\} ; \mathrm{n}=\mathbf{1 , 2 , 3}, \cdots \mathrm{Z}
$$

\section{b) RGB Values enhancement}

In the developed technique, the RGB is possessed by the RGB is changed in a way to indicate it highly identifiable by increasing the information content in the input image. By exploiting the optimization algorithm aforesaid approach is carried out. When considering UI, the green and blue colors are the dominant colors, hence the channels are modified to decrease the water effect o the image preceding commence the advancement procedure. Each RGB channel needs to attune to a favored Median value. To obtain this, every channel is altered by evaluating a constant positive or negative value using the optimization algorithm. In order to enhance the RGB values, the eq. (2), (3), and (4) are used.

$$
\begin{aligned}
& R^{\prime}=M_{1}+R \\
& G^{\prime}=M_{2}+G \\
& B^{\prime}=M_{3}+B
\end{aligned}
$$

Whereas, $R, G$, and $B$ indicates real $\mathrm{RGB} ; R^{\prime}, G^{\prime}$, along with $B^{\prime}$ indicates equal values once subsequent to the enhancement, and $M_{1}, M_{2}, M_{3}$ are presumed as the constant values may be negative else positive that are deployed to modify the target values of the blue channel beside the mean values of the green and red. Subsequent to, for the RGB values, the Median beside SD is calculated by exploiting the eq. (5) and (6),

$$
\begin{aligned}
& \overline{\mathrm{U}}=\frac{\left(\sum \mathrm{U}(\mathrm{x}, \mathrm{y})\right)}{\mathrm{n}} \\
& \mathrm{SD}=\sqrt{\frac{\sum(\mathrm{U}-\overline{\mathrm{U}})^{2}}{\mathrm{n}-\mathbf{1}}}
\end{aligned}
$$

Wherein, $U$ indicates input image at $256 \times 256$ pixels, $\bar{U}$ indicates subjected $\mathrm{RGB}$ value median, and subsequently $n$ indicates the aggregate count of input images. Therefore, the SD, as well as median, is estimated for the green, red, besides blue color. Subsequently, employ the developed technique 
contributed to the ordinary images that are improved by exploiting the advanced optimization method. Then the improvement transformation is performed to each pixel at the position $(x, y)$ as Eq. (7).

$$
g(x, y)=m(x, y)^{p}+[f(x, y)-q * m(x, y)]\left[s \frac{D}{\sigma(x, y)+r}\right]
$$

Wherein, $f(x, y)$ and $g(x, y)$ indicates the pixels' gray-level intensity on the input and output images, respectively, grounded at the position $(x, y)$. For the entire image, the parameters $p, q, r$, and $s$ are taken into consideration and are indicted as real positive numbers. $D$ indicates the global median for the entire image and it is calculated using eq. (8). For the neighborhood centered at $(x, y)$ the gray level mean estimated is given by $\mathrm{m}(\mathrm{x}, \mathrm{y})$.

$$
\mathrm{D}=\frac{\left(\sum \mathrm{U}\right)}{\mathrm{n}}
$$

Wherein, $D$ indicates global median for the entire image, $U$ indicates subjected UI. $\sigma(x, y)$, the graylevel SD computed at neighborhood grounded at $(x, y)$, and it is computed using Eq. (9),

$$
\sigma(\mathrm{x}, \mathrm{y})=\sqrt{\frac{1}{\mathrm{n}} \sum_{\mathrm{x}=\mathbf{0}}^{\mathrm{n}} \sum_{\mathrm{y}=\mathbf{0}}^{\mathrm{n}}(\mathrm{f}(\mathrm{x}, \mathrm{y})-\mathrm{m}(\mathrm{x}, \mathrm{y}))^{2}}
$$

Moreover, by exploiting the advanced optimization algorithm, the 4 parameters $p, q, r$, and $s$ are optimized.

\section{c) Feature Extraction}

Feature extraction is the process to modify the object-recognized image into the feature series. The SURF feature, Hurst Exponent, Harris Feature, Skewness, Kurtosis, SD, Correlation, Variance, Mean, contrast, Entropy, and Homogeneity are used to describe the image content.

\section{i)Robust Features of Speeded-Up}

It is a local feature detector and a descriptor that is used for the tasks. The SURF features are exploited for the shifting invariant, scaling, and rotation, as well as partially invariant to enlightenment and as well affine transformation, are stated in Eq. (10).

$$
\mathrm{H}(\mathrm{x}, \sigma)=\left(\begin{array}{ll}
\mathrm{L}_{\mathrm{xx}}(\mathrm{X}, \sigma) & \mathrm{L}_{\mathrm{xy}}(\mathrm{X}, \sigma) \\
\mathrm{L}_{\mathrm{xy}}(\mathrm{X}, \sigma) & \mathrm{L}_{\mathrm{yy}}(\mathrm{X}, \sigma)
\end{array}\right)
$$

wherein, $\mathrm{L}_{\mathrm{XX}}(\mathrm{X}, \sigma)$ indicates the Gaussian convolution $2^{\text {nd }}$ order derivative with the image, and likewise for $\mathrm{L}_{\mathrm{xy}}(\mathrm{X}, \sigma)$ and $\mathrm{L}_{\mathrm{yy}}(\mathrm{X}, \sigma)$.

\section{ii) Harris Feature}

In an image, the features are found using the Haris corner detector that indicates as a mathematical formulation and it is stated using eq. (11). Wherein intensity change aspired at the shift is termed as the $E(u, v), \mathrm{w}(\mathrm{x}, \mathrm{y})$ signifies the window function, $\mathrm{I}(\mathrm{x}+\mathrm{u}, \mathrm{y}+\mathrm{v})$ indicates the shifted intensity, and subsequently $I(x, y)$ indicates intensity.

\section{iii) Hurst Exponent}

$$
E(u, v)=\sum_{x, y} w(x, y)[I(x+u, y+v)-I(x, y)]^{2}
$$

This is a self-similarity gauge, predictability as well as longer-range reliance level in a timeseries. It is similarly a smoothness gauge of a fractal time series based on asymptotic conduct of the re-scaled range for the process in line with the Hurst's sum up the time series formulation, based on Eq. (12) $H$ is characterized.

$$
F_{3}=H=\frac{\log \left(\frac{R}{S}\right)}{\log (T)}
$$

Wherein, $R / S$ indicates equivalent re-scaled range value, $T$ indicates data sample duration. $R$ indicates a contrast between the minimum and maximum deviation from the mean, when, $S$ indicates the SD. So that, eq. (13) indicates the Hurst Exponent wherein, $c$ indicates a constant beside to $H$ indicates Hurst Exponent.

$$
\left(\frac{\mathrm{R}}{\mathrm{S}}\right)_{\mathrm{T}}=\mathrm{c} * \mathrm{~T}^{\mathrm{H}}
$$




\section{iv) Mean ( $M)$}

$M$ of an image is computed using by augmenting the image's whole values of the pixel which are divided using the aggregate number of pixels in the image and it is shown in Eq. (14).

$$
M_{X}=M=\left(\frac{1}{m \times n} \sum_{x=0}^{m} \sum_{y=0}^{n} f(x, y)\right)
$$

\section{v) Standard Deviation}

It is presumed as the $2^{\text {nd }}$ central moment defined realistic population PD and act as a homogeneity gauge. The enhanced intensity level and for the image better edge contrast is referred in eq. (15) and its superior value is indicated in eq. (15).

$$
\mathrm{M}_{\mathrm{y}}=\mathrm{SD}(\sigma)=\sqrt{\left(\frac{1}{\mathrm{~m} \times \mathrm{n}}\right) \sum_{\mathrm{x}=\mathbf{0}}^{\mathrm{m}} \sum_{\mathrm{y}=\mathbf{0}}^{\mathrm{n}}(\mathrm{f}(\mathrm{x}, \mathrm{y})-\mathrm{M})^{\mathbf{2}}}
$$

\section{vi) Kurtosis}

The haphazard variables shape PD is referred to using a parameter called kurtosis. $X$ indicates a haphazard variable, and the kurtosis is indicated as $K_{u r t}(X)$ and it is shown in eq. (16).

$$
\mathrm{K}_{\text {urt }}(\mathrm{X})=\left(\frac{1}{\mathrm{~m} \times \mathrm{n}}\right) \frac{\sum(\mathrm{f}(\mathrm{x}, \mathrm{y})-\mathrm{M})^{4}}{\mathrm{SD}^{4}}
$$

\section{vii) Skewness $\left(S_{k}\right)$}

It is referred to as asymmetry gauge or symmetry short. The eq. (17) states the haphazard variable $(X)$ skewness.

$$
\mathrm{S}_{\mathrm{k}}(\mathrm{X})=\left(\frac{\mathbf{1}}{\mathrm{m} \times \mathrm{n}}\right) \frac{\sum(\mathrm{f}(\mathrm{x}, \mathrm{y})-\mathrm{M})^{\mathbf{3}}}{\mathrm{SD}^{\mathbf{3}}}
$$

\section{viii) $\operatorname{Variance}(V)$}

The variance is referred to as the measure and it is stated in eq. (18), which implies as of the mean how much of grayscale is varying.

$$
\mathrm{V}=\sum_{\mathrm{y}} \sum_{\mathrm{x}} \mathrm{f}(\mathrm{x}, \mathrm{y}) \mathrm{f}(\mathrm{x}, \mathrm{y})-\mu^{2}
$$

\section{ix) Contrast ( $C$ )}

The contrast returns the contrast of intensity measure between a pixel as well as its nearby through the entire image. In addition, the contrast is zero for a continual image and it is stated in eq. (19), wherein, the contrast is represented as $C$ and $f(x, y)$ indicates pixel at position $(x, y)$

$$
\mathrm{C}=\sum_{\mathrm{x}, \mathrm{y}}|\mathrm{x}-\mathrm{y}|^{2} \mathrm{P}(\mathrm{x}, \mathrm{y})
$$

\section{x) Correlation}

Eq, (20) indicates the correlation features, as well as the spatial dependencies among the pixels.

\section{xi) Entropy ( $E$ )}

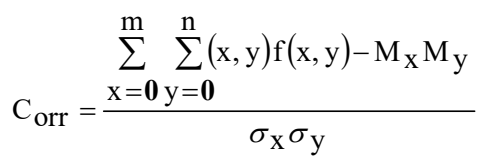

Eq. (21) indicates the return of the squared feature's total on the GLCM. It is considered as the firmed image.

$$
E=\sum_{x, y} f(x, y)^{2}
$$

\section{xii) Homogeneity $(H)$}

Eq. (22) states the proffers back a value that measures the distribution features closeness on GLCM to the diagonal. 


$$
H=\sum_{x, y} \frac{f(x, y)}{1+|x-y|}
$$

\section{d) Feature Reduction}

After completing the extraction of features, by exploiting the enhanced PCA approach the features are minimized. PCA indicates as a technique that is generally exploited aspired at feature minimization. It is used to identify the pattern on the data and emphasizes the resemblance besides with difference. The principal advantage of PCA is that formerly the pattern is obtained, and subsequently, the number of dimensions of the data-set is reduced. Moreover, the enhanced PCA is exploited. Enhanced PCA is an improved version of PCA whereas each image is divided nto the number of sub-block images and after that PCA is allocated for each sub-block image. The EPCA steps are expressed as stated as follows:

a) Identifies the dataset.

b) As stated in eq. (23), (24), and (25), Gaussian Kernel is implemented.

$$
\begin{aligned}
& \text { temp }=\operatorname{sum}\left(\left(\text { data } \_ \text {in }(:, \text { row })-\text { data } \_i n(:, \text { col })\right) \cdot \wedge 2\right) \text {; } \\
& \mathrm{k}\left(\mathrm{x}_{\mathrm{i}}, \mathrm{x}\right)=\exp \left(-\frac{\left\|\mathrm{x}_{\mathrm{i}}-\mathrm{x}\right\|^{2}}{2}\right) \\
& \mathrm{k}^{\prime}=\mathrm{k}+\mathrm{k} !
\end{aligned}
$$

c): CM is computed. As stated in eq. (26), the covariance is measured in multi-dimension.

$$
\operatorname{Cov}(\mathrm{x}, \mathrm{y})=\frac{\sum_{\mathrm{i}=\mathbf{1}}^{\mathrm{N}}\left(\mathrm{X}_{\mathrm{i}}-\overline{\mathrm{X}}\right)\left(\mathrm{Y}_{\mathrm{i}}-\overline{\mathrm{Y}}\right)}{\mathrm{n}-\mathbf{1}}
$$

In a $\mathrm{CM}$, if all the non-diagonal modules have a positive value subsequently it refers to the $X, Y, Z$ variable increases together.

d): CM's Eigen Vectors beside to Eigen Values are computed. Here, from the CM, calculate an Eigenvalue beside the Eigenvector.

e):Interpret this data into modules.

\section{e) ANFIS classifier for Classification}

The $w\left(\vec{x}_{1}\right), w\left(\vec{x}_{2}\right), w\left(\vec{x}_{n}\right)$ represents the maximized features, which is extricated from the optimization of enhanced BA [6]. Here, the ANFIS classifier [7] is exploited for the classification procedure. It consists of five node layers. In these five layers, the first with the fourth layers has adaptive nodes whereas the second third, and the fifth layer possess fixed nodes. The ANFIS is exploited to categorize the features. The main basic rules of the ANFIS model in the form. If $w\left(\vec{x}_{1}\right)$ is $A_{i}, w\left(\vec{x}_{2}\right)$ is $B_{i}$, and $w\left(\vec{x}_{n}\right)$ is $C_{i}$ and subsequently, it is stated in eq. (27).

$$
\text { Rules }_{\mathrm{i}}=\mathrm{a}_{\mathrm{i}} * \mathrm{w}\left(\overrightarrow{\mathrm{x}}_{\mathbf{1}}\right)+\mathrm{b}_{\mathrm{i}} * \mathrm{w}\left(\overrightarrow{\mathrm{x}}_{2}\right)+\mathrm{c}_{\mathrm{i}} * \mathrm{w}\left(\overrightarrow{\mathrm{x}}_{\mathrm{n}}\right)+\mathrm{f}_{\mathrm{i}}
$$

wherein, $A_{i}, B_{i}$, and $C_{i}$ defines fuzzy series, $w\left(\vec{x}_{1}\right), w\left(\vec{x}_{2}\right), \& w\left(\vec{x}_{n}\right)$ indicates the inputs, Rules indicates throughput amid the particular fuzzy area through the fuzzy rule, $a_{i}, b_{i}$, besides to the $c_{i}$ indicate smodeling parameters that are decided via the training model.

1st Layer:Eq. (28) denotes each node $i$ of this particular layer comprises a square node that possesses a node function.

$$
\left.\mathrm{O}_{1, \mathrm{i}}=\mu_{\mathrm{Ai}}\left(\mathrm{w}\left(\overrightarrow{\mathrm{x}_{1}}\right)\right), \mathrm{O}_{\mathbf{1}, \mathrm{i}}=\mu_{\mathrm{Bi}}\left(\mathrm{w}_{\left(\overrightarrow{\mathrm{x}_{2}}\right.}\right)\right), \mathrm{O}_{\mathbf{1}, \mathrm{i}}=\mu_{\mathrm{Ci}}\left(\mathrm{w}\left(\overrightarrow{\mathrm{x}_{\mathrm{n}}}\right)\right)
$$

Generally, $\mu_{A i}\left(w\left(\overrightarrow{x_{1}}\right)\right), \mu_{B i}\left(w\left(\overrightarrow{x_{2}}\right)\right)$, and $\mu_{C i}\left(w\left(\overrightarrow{x_{n}}\right)\right)$ indicates select to be bell-shaped with optimum parallel to one and least amount equal to zero and are explained in eq. (36). Wherein, $o_{i}, p_{i}$, $q_{i}$ indicates a set of parameters and is also explained as fundamental parameters.

$$
\mu_{\mathrm{Ai}}\left(\mathrm{w}\left(\overrightarrow{\mathrm{x}_{1}}\right)\right)=\mu_{\mathrm{Bi}}\left(\mathrm{w}\left(\overrightarrow{\mathrm{x}_{2}}\right)\right)=\mu_{\mathrm{Ci}}\left(\mathrm{w}\left(\overrightarrow{\mathrm{x}_{\mathrm{n}}}\right)\right)=\frac{1}{1+\left[\left(\frac{\mathrm{x}-\mathrm{o}_{\mathrm{i}}}{\mathrm{p}_{\mathrm{i}}}\right)^{2}\right]^{\mathrm{q}_{\mathrm{i}}}}
$$

$2^{\text {nd }}$ Layer:Each node comprises a Circle Node $(\mathrm{CN})$ labeling which multiplies the received signals and the product is transmitted out that is stated in eq. (30). Each node throughput denotes a rule's Firing Strength (FS).

$$
\mathrm{O}_{2, \mathrm{i}}=\mathrm{wt}_{\mathrm{i}}=\mu_{\mathrm{Ai}}\left(\mathrm{w}\left(\overrightarrow{\mathrm{x}_{\mathbf{1}}}\right)\right) \times \mu_{\mathrm{Bi}}\left(\mathrm{w}\left(\overrightarrow{\mathrm{x}_{2}}\right)\right) \times \mu_{\mathrm{Ci}}\left(\mathrm{w}\left(\overrightarrow{\mathrm{x}_{\mathrm{n}}}\right)\right), \quad \mathrm{i}=\mathbf{1}, \mathbf{2}(30)
$$


$3^{\text {rd }}$ Layer: Each node holds a CN indicated $N$. The $i^{\text {th }}$ node calculates the ratio of the $i^{\text {th }}$ rule's FS to the entire of each rule's FS and it is stated in eq. (31).

$$
\mathrm{O}_{3, \mathrm{i}}=\overline{\mathrm{wt}}_{\mathrm{i}}=\frac{\mathrm{wt}_{\mathrm{i}}}{\left(\mathrm{wt}_{\mathbf{1}}+\mathrm{wt}_{\mathbf{2}}\right)}, \quad \mathrm{i}=\mathbf{1 , 2}
$$

$4^{\text {th }}$ Layer:Eq. (32) states the each node $i^{\text {th }}$ possess a square node using a node function.

$$
\mathrm{O}_{\mathbf{4}, \mathrm{i}}=\overline{\mathrm{wt}}_{\mathrm{i}} \text {.Rules } \mathrm{i} \quad \mathrm{i}=\mathbf{1 , 2}
$$

Wherein $w t_{i}$ indicates the layer 3 's output and $a_{i}, b_{i}, c_{i}, f_{i}$ represents parameters. Moreover, the Parameters of this layer are referred to as the following parameters.

$5^{\text {th }}$ Layer: The single node of this layer possess a CN called $\Sigma$ which measures the whole throughput as the review of whole receiving signals and it is represented in Eq. (33) and (34).

$$
\begin{gathered}
\mathrm{O}_{5, i}=\sum_{\mathrm{i}} \overline{\mathrm{wt}}_{\mathrm{i}} \mathrm{Rules}_{\mathrm{i}}=\frac{\sum_{\mathrm{i}} \mathrm{wt}_{\mathrm{i}} \mathrm{Rules}_{\mathrm{i}}}{\sum_{\mathrm{i}} \mathrm{wt}_{\mathrm{i}}} \\
\mathrm{n}\left(\mathrm{o}_{\mathrm{i}}^{\mathrm{f}}\right)=\overline{\mathrm{wt}}{ }_{\text {Rules }}+\overline{\mathrm{wt} R u l e s} \mathbf{2}
\end{gathered}
$$

For that reason, the ANFIS is used to classify the obtained feature, and subsequently, the classified feature is indicated as $n\left(O_{i}^{f}\right)$. Subsequently, the formerly-tries to threshold value $\omega$ together with the NN upshot is $(Y)$. The NN output $Y$ better to the threshold value $\omega$ means, the presented input image is acknowledged and $Y$ minimum threshold value $\omega$ indicates the image is not acknowledged.

\section{Proposed improved BAT Algorithm}

In this paper, the Enhanced Bat algorithm is proposed [6] and it is performed by enhancing the relationship associated with the location and each Bat speed as an optimization solution. To find the solutions, the Bat algorithm is considered a rapid approach. The eq. (35) is attained as an enhanced location alters association for each bat as an optimization solution.

$$
x_{i}^{t}=x_{*}+v_{i}^{t}
$$

The bats fly pattern change permits each bat to move to the best location. This alters aids the approach to converge. The issue with this association is that the approach is attentive in the local optima. Accordingly, a measure should be formulated, therefore; the approach verifies the whole search space to identify the important solution. In this paper, by augmenting an arbitrary constant the mutation operator works in the search space range to the present location matrix of each bat and the best solution.

The whole search space is verified by this operator that approaches extremely ability to find the optimal position for issues. To various population members, the mutation operator is arbitrarily used as well as a definite proportion of the location matrix in every solution. Each bat location is a function of the best position as well as velocity as seen in eq. (35).

Since each bat speed attains " 0 " because of the optimal deviation after the prey hunting, this though cannot deviate from the best location of any solution. Hence, the

The coefficient of self-matching $w$ is exploited to " 0 " the velocity vector while attaining the original optimum. Therefore, eq. (36) associated with the velocity vector of each bat is enhanced as eq. (37).

$$
\begin{aligned}
& v_{i}^{t}=v_{i}^{t-1}+\left(x_{i}^{t-1}-x *\right) f_{i} \\
& v_{i}^{t}=w \cdot\left(v_{i}^{t-1}+\left(x_{i}^{t-1}-x *\right) f_{i}\right)
\end{aligned}
$$

The coefficient $w$ is optimized hence the location vector does not deviate while it attains the position. This coefficient is flexible and variable at the time of the approach execution at each step.

The coefficient optimal side will lead to "0". The relationship $w$ is in the form of Eq. (38).

$$
\mathrm{w}=\mathrm{x} * \mathrm{i}-\mathrm{x} * \mathrm{i}-1
$$

wherein $x_{* i}$ indicates the current best location vector and $x_{* i-1}$ indicates the best location vector in the preceding iteration.

\section{Experimental Analysis}

In this section, the experimental analysis of the proposed and conventional models has been experimented. Here, the underwater images were attained from the publicly present websites. Here, the 
database was created on the basis of the obtained images. Here, the database comprises two kinds of images such as shallow water images as well as deep water images.

Fig 2 shows the performance analysis of the proposed and conventional models. Here, the analysis is performed on the basis of both the positive and negative measures. From the fig, it is evident the proposed IBA-ANFIS performance is better than the conventional models in terms of both the positive as well as negative metrics.

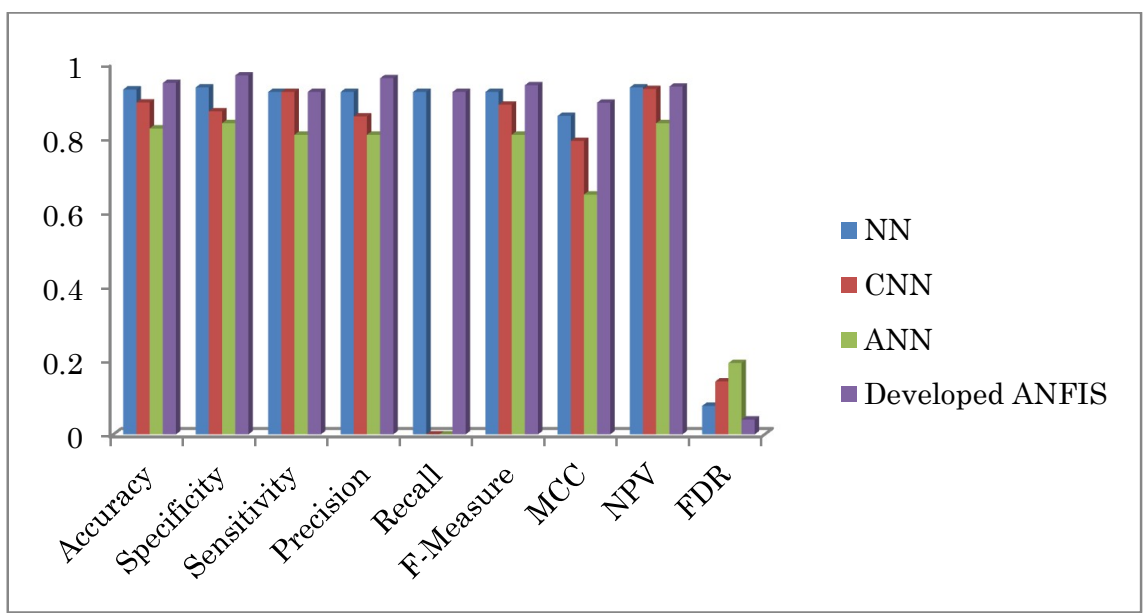

Fig. 2. Performance analysis of the proposed and conventional models

\section{Conclusion}

In this paper, a capable optimization technique named IBA for the enhancement as well as the UI classification was developed. The performance of the developed model was examined in the UI obtained from the database. The performance analysis of the proposed model was shown that the enhancement of UI, as well as the classification model, subjected an outstanding sensitivity rate, specificity as well as accuracy recall, precision, MCC, NPV, FDR as well as F-measure. The performance evaluation of the proposed and conventional model was exhibited and it has obtained maximum sensitivity, accuracy as well as specificity, however, the conventional method attainsless. Therefore, the overall analysis exhibits that the proposed method performance is better and accurate while comparing with the conventional techniques regarding the underwater images.

\section{Compliance with Ethical Standards}

Conflicts of interest: Authors declared that they have no conflict of interest.

Human participants: The conducted research follows the ethical standards and the authors ensured that they have not conducted any studies with human participants or animals.

\section{References}

[1] M. Machado Dos Santos, G. G. De Giacomo, P. L. J. Drews and S. S. C. Botelho, "Matching Color Aerial Images and Underwater Sonar Images Using Deep Learning for Underwater Localization," IEEE Robotics and Automation Letters, vol. 5, no. 4, pp. 6365-6370, Oct. 2020.

[2] C. Li, S. Tang, H. K. Kwan, J. Yan and T. Zhou, "Color Correction Based on CFA and Enhancement Based on Retinex With Dense Pixels for Underwater Images," IEEE Access, vol. 8, pp. 155732-155741, 2020.

[3] Y. Wang, W. Song, G. Fortino, L. Qi, W. Zhang and A. Liotta, "An Experimental-Based Review of Image Enhancement and Image Restoration Methods for Underwater Imaging," IEEE Access, vol. 7, pp. 140233$140251,2019$.

[4] M. Yang, J. Hu, C. Li, G. Rohde, Y. Du and K. Hu, "An In-Depth Survey of Underwater Image Enhancement and Restoration," IEEE Access, vol. 7, pp. 123638-123657, 2019.

[5] G. Hou, X. Zhao, Z. Pan, H. Yang, L. Tan and J. Li, "Benchmarking Underwater Image Enhancement and Restoration, and Beyond," IEEE Access, vol. 8, pp. 122078-122091, 2020.

[6] Minghai YuanYadong LiFengque Pei,"Research on intelligent workshop resource scheduling method based on improved NSGA-II algorithm", Robotics and Computer-Integrated Manufacturing, 2 March 2021. 
Underwater Image Enhancement Using Improved Bat Algorithm

[7] Hussam Eldin ElzainSang Yong ChungMohamed Hassan,"ANFIS-MOA models for the assessment of groundwater contamination vulnerability in a nitrate contaminated area", Journal of Environmental Management,24 February 2021.

[8] Santosh Kumar B. P,Venkata Ramanaiah K,"An Efficient Hybrid Optimization Algorithm for Image Compression",Multimedia Research,vol. 2, no. 4, October 2019.

[9] Nipanikar S I,Hima Deepthi V,"Enhanced Whale Optimization Algorithm and Wavelet Transform for Image Steganography", Multimedia Research, vol 2, no. 3, July 2019. 